\title{
CORTISONA * EN PREMATUROS
}

\author{
Prof. JULIO MENEGHELLO y Drs. OLGA ROYO \\ y ALEJANDRO MANTEROLA \\ Cátedra Extraordinaria de Pediaris dal Pró. Julio Meneghello. \\ Hospital "Manuel Arriarán".
}

Aunque no hay evidencias exactas que indiquen en forma incontrovertible un estado fisiológico de hipoadrenalismo en el recién nacido, las investigaciones citológicas e histoquímicas han descubierto ciertas diferencias significativas entre la apariencia histológica y la actividad suprarrenal en el recién nacido normal y en el prematuro $1 \cdot z$. La corteza neonatal está formada por 2 zonas, una delgada, que es periferica y otra hipertrófica, corteza fetal propiamente dicha, situada entre esta y al zona medular. Los estudios citológicos de Swinyard, permiten suponer que el estroma de la corteza fetal sea la base de la zona reticular que aparece en el 3.er mes de la vida extrauterina.

Para Moore y Wells" la corteza fetal hipertrofiada representaría una respuesta a la influencia hormonal materna, la que actuaría a través de la hipófisis fetal; una vez verificado el parto, cesa el estimulo y la corteza, con la fuga de las hormonas maternas, comienza una rápida involución. Concomitantemente con esto se produciría una hipofunción suprarrenal de grado variable, en la primera quincena de la vida en lo que se refiere a hormonas de retención, y el recién nacido, especialmente el prematuro, debe afrontar las emergencias a expensas de su propia corteza, cuya capacidad puede no ser suficiente para responder en forma adecuada a la agresion.

Por otra parte la hipoglicemia fisiológica y los elevados recuentos de eosinófilos del recién nacido también han sido invocados en apoyo de esta tesis ${ }^{1}$. El uso de estímulos indirectos como la epinefrina - bien el ACTH, es capaz de producir eosinopenia en el recién nacido, y estaría en directo relación con el peso, edad y grado de madurez del niño. Los trabajos de Jailer 5 sugieren que la incapacidad de algunos niños para hacer frente a condiciones que les exigen máximo esfuerzo, podría explicarse por falta de desarrollo del mecanismo adrenopituitario en el prematuro y algunos niños de término.

* Cortone Werck.
Lanmann "al dosificar las corticoides formaldehidógenos y estudiando la respuesta frente a la administración de ACTH no comprueba la insuficiencia suprarrenal en el prematuro.

El éxito alcanzado con el empleo de las hormonas corticales en diferentes cuadros clínicos del adulto y del niño, y el mejor conocimiento de la fisiología de la corteza del recién nacido y del niño mayor, han hecho incrementar el uso de las hormonas corticales en Pediatría.

Niños sometidos a stress por diferentes causas, prematuros con fibroplasia retrolental $\mathrm{y}$ otros con defectos metabólicos han sido estudiados desde el punto de vista de la acción hormonal, y así vemos que hace más o menos dos años aparecieron las primeras comunicaciones médicas acerca del uso de ACTH y Cortisona en prematuros.

Levine " analizó la respuesta clínica y metabólica en 17 prematuros tratados con ccrticotrofina y encontró que en ellos se corregía la tirosiluria determínada por la dieta libre de leche de vaca sin agregado de vitamina $C$ (substancia que corrige este trastornos metabólico). También la cortisona corrigió la anormal excreción de productos intermediarios de la tirosina en la orina de 3 prematuros, en una serie de 8 tratados con 300 o más miligramos de cortisona como dosis total. Además se observó que la curva de peso se modificaba, en el sentido de disminuír el aumento ponderal durante el empleo del medicamento, en relación al período anterior. En algunos casos se comprobó que hubo pérdida de peso durante el tratamiento.

Con posterioridad se observó marcado aumento de peso, de modo que el promedio de la ganancia ponderal durante $\mathrm{y}$ después, resultó más elevado que el promedio que se había establecido para la etapa previa al uso de ACTH. Durante los dias que se usó la hormonoterapia se advirtió además aumento de la actividad muscular, del apetito y de la intensidad de] llanto.

Silvermann y cols. ${ }^{8}$ trataron 19 prematuros afectados de fibroplasia retrolental 
CLATSR NE 1

EDAD AL INICIAR LA OBSERVACION PREMATUROS TRATADOS $Y$ NO TRATADOS CON CORTISONA.

\begin{tabular}{|c|c|c|}
\hline & $\begin{array}{c}\text { Tratados } \\
\text { días }\end{array}$ & $\begin{array}{c}\text { No tratados } \\
\text { dias }\end{array}$ \\
\hline & $\begin{array}{r}13 \\
7 \\
6 \\
11 \\
y \\
7 \\
5 \\
17 \\
11 \\
9\end{array}$ & $\begin{array}{r}13 \\
7 \\
4 \\
11 \\
3 \\
7 \\
5 \\
16 \\
13 \\
113\end{array}$ \\
\hline Promedia: & $9 ., i$ & 9.5 \\
\hline
\end{tabular}

con ACTH, con el propósito de actuar sobre este proceso. Comprobaran aumento del apetito y de la actividad muscular, con retardo o lentitud del ascenso ponderal durante los dias de tratamiento y mayor progreso al rebajar la dosis a la mitad o suspender el empleo. La aceleración en el engorde se mantuvo en líneas generales, durante 2 semanas en la mayor parte de los niños, después de suspendido. Además, comprobaron detención del crecimiento esquelético durante los periodos de tratamiento con normalización posterior.

En nuestro trabajo relatamos la experiencia que nos hemos formado con el empleo de Cortisona en el prematuro sano. Estudiamos las reacciones clínicas expresadas en curva de peso, modificaciones del apetito, actividad, hidratación $y$ complicaciones infecciosas. En la revisión bibliográfica no hemos encontrado referencias al uso exclusivo de Cortisona en prematuros.

\section{PLAN DE TRATAMIENTO}

Durante 20 días se dió Cortisona a 10 prematuros hospitalizados en el Pabellón especializado del Hospital Manuel Arriarán, entre Marzo y Agosto de 1952. De ellos, 7 recibieron la hormona por vía oral y 3 por via inyectable. La dosis fué uniforme: 12 mgrs. diarios repartidos en 2 porciones durante 10 días. En los restantes 10 días se dieron $6 \mathrm{mgrs}$. diarios. La dosis total para cada niño fué de 180 mgrs.

\section{MATERIAL Y MÉTODO}

Al iniciar el tratamiento el peso promedio de los prematuros tratados fué de 1.727 grs. y su edad promedio de 9,5 días
( EDPRO Kis 2

PESO A LOS 9.5 DIAS DE VIDA. PREMATUROS TRATADOS Y NO TRATADOS CON CORTISONA.

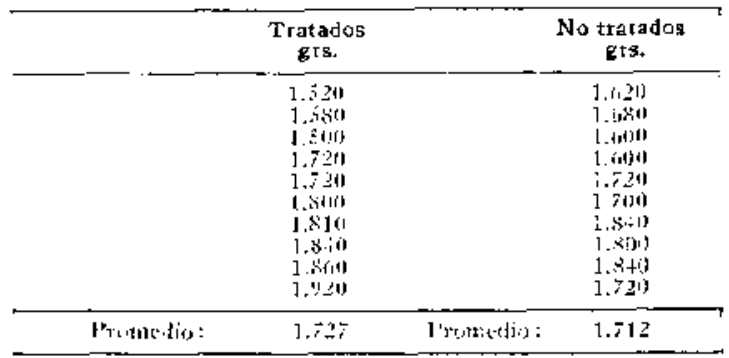

(cuadros 1 y 2). El período de observación intrahospitalario previo al tratamiento fluctuó entre 4 y 17 días. Todos fueron sometidos a igual tratamiento: incubadora Armstrong, penicilina oral los primeros 10 días de estada; Vitamina $C, 50$ mgrs. diarios, alimentación con una mezela de Eledón al $12 \%$ con 3\% de dextromatosa y $5 \%$ de azúcar. Además las condiciones ambientales de temperatura $y$ humedad fueron similares. Todos fueron tratados con igual rutina de cuidados, efectuadas por una planta estable de médicos, enfermera y auxiliares.

El grupo de control fué formado por prematuros ingresados dentro del mismo año y tratados en la misma forma, excepto en que no se les dió Cortisona. Se seleccionaron estos casos controles, buscando para cada niño, uno que le hiciera pareja de acuerdo con el sigujente criterio de comparación:

a) Peso de nacimiento (cuadro 3 ) cuyo promedio para el grupo de tratados con Cortisona fué de 1.944 grs. y para los no tratados fué de $2.000 \mathrm{grs}$.

b) Peso al iniciar la observación (cuadro 2): En los nin̆os que recibieron Cortisona alcanzo en promedio 1.727 grs. y en los controles 1.712 grs.

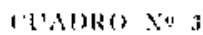

PESO DE NACIMIENTO. PREMATUROS TRATADOS Y NO TRATADOS CON CORTISONA.

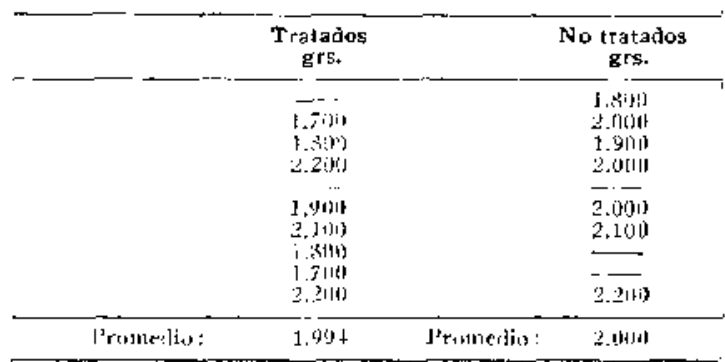


CTADRO Se 4

ALIMENTACION RECIBIDA SEGUN EDAD. PREMA. TUROS TRATADOS Y NO TRATADOS CON CORTISONA.

\begin{tabular}{|c|c|c|}
\hline $\begin{array}{c}\text { Edad en dias } \\
\text { (promedio) }\end{array}$ & Aporte & $\begin{array}{l}\text { calóricu } \times \mathbf{K g} \text {. peso } \\
\text { (promedio) }\end{array}$ \\
\hline & Tratados & No tratados \\
\hline $\begin{array}{r}9,5 \\
14,5 \\
19,5 \\
24,5 \\
29,5 \\
34.5 \\
39,5\end{array}$ & $\begin{array}{r}46,8 \\
97,6 \\
107,2 \\
109,8 \\
321,4 \\
1.31,5 \\
1.39,0\end{array}$ & $\begin{array}{r}41,2 \\
3111,01 \\
115.9 \\
117,3 \\
120,5 \\
1+11.5 \\
1-1,3.5\end{array}$ \\
\hline
\end{tabular}

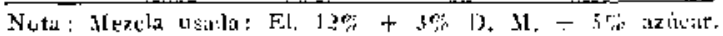

c) La edad promedio al iniciar la observación (cuadro 3 ) fué de 9.5 dias en ambos grupos.

d) La alimentación fué semejante en ambos grupos (cuadros 4 y 5 ) a base de Eledón al $12 \%$ con agregado de $8 \%$ de hidratos de carbono, durante el período que duró la observación comparativa.

e) El tiempo de observación comparativa de los pacientes fué de 30 días en ambos grupos.

\section{RESULTADOS}

Analizaremos en forma separada el efecto del tratamiento sobre la curva de peso, sobre el apetito, la actividad y complicaciones.

a) Sobre la curva de peso: Durante el período de tratamiento (20 días) los niños tratados con Cortisona tuvieron en promedio una ganancia en peso de $181 \mathrm{grs}$. $160 \mathrm{grs}$. en los primeros 10 días y 121 en la segunda mitad). En un periodo igual el grupo control tuvo un aumento de 246 grs. ( 85 grs, en los primeros 10 días y 161 grs. en la segunda mitad). Entre el $20^{\circ}$ y $30^{\circ}$ dia de observación el progreso fué de 283 y 305 grs. respectivamente. En los 30 días de observación, el ascenso en los tratados con Cortisona, fué de $464 \mathrm{grs}$. $\mathrm{y}$ en los niños no tratados fué de $\mathbf{5 5 1}$ grs.

CLADHO NI!

AFECCIONES INTERCURRENTES OBSERVADAS ENTRE EL 9.5 DIA Y EL 29.5 DIA DE VIDA. PRE. MATUROS TRATADOS $Y$ NO TRATADOS CON CORTISONA.

\begin{tabular}{|c|c|c|}
\hline \multirow[b]{2}{*}{ Diagnósticos } & \multicolumn{2}{|c|}{ Frecuencia } \\
\hline & Tratados & No tratados \\
\hline Diasre:ts nubosinturtáticas & 2 & $\frac{2}{2}$ \\
\hline $\begin{array}{l}\text { Mispepias } \\
\text { Mromputis: }\end{array}$ & 2 & 2 \\
\hline 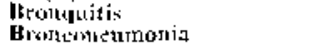 & 3 & 2 \\
\hline $\begin{array}{l}\text { Brortinuetumosia } \\
\text { Alelect:ses }\end{array}$ & - & $\frac{1}{1}$ \\
\hline Alelect:1s: & $\bar{n}$ & 1 \\
\hline $\begin{array}{l}\text { Abstitsus } \\
\text { Otitis }\end{array}$ & $\begin{array}{l}2 \\
1\end{array}$ & $=$ \\
\hline
\end{tabular}

$$
\text { (CADRO NE: }
$$

ALIMENTACION RECIBIDA SEGUN EDAD. PREMATUROS TRATADOS Y NO TRATADOS CON CORTISONA.

\begin{tabular}{|c|c|c|}
\hline $\begin{array}{l}\text { Edad en dias } \\
\text { (promedio) }\end{array}$ & Yolumen & $\begin{array}{l}\text { diario } x \\
\text { (promedio) }\end{array}$ \\
\hline & Tratados & No Iratados \\
\hline 9.5 & 107.1 & 97.8 \\
\hline 34.5 & 1196 & 122.0 \\
\hline 19.5 & $1,30.7$ & $1+1, \overline{7}$ \\
\hline 24,3 & 135.4 & 143.0 \\
\hline 29,3 & $14 k, n$ & 159.4 \\
\hline 34.5 & 361,7 & 171.3 \\
\hline 31,5 & $1>0$, in & 174.6 \\
\hline
\end{tabular}

El detalle de estas cifras se presenta en los cuadros 6 y 7 que permitieron construír los gráficos 1 y 2 . Las diferencias comprobadas no son significativas.

b) Sobre el apetito: No observamos mayor avidez para alimentarse en los prematuros tratados con Cortisona que en los que no lo fueron. Además, en 7 de los niños tratados no se advirtió cambio del apetito, durante la administración de la hormona, en comparación al que habian demostrado previamente ni después del uso de ella. En 2, el apetito pareció aumentar y este aumento se mantuvo posteriormente. En 1 niño se observó franca inapetencia hacia el final de la serie de inyecciones, pero este trastorno puede explicarse por un proceso infeccioso que sufrió el paciente en este período. En el grupo control el apetito fué considerado como muy bueno en 3 casos, como bueno en los 7 restantes.

c) Sobre la actividad: Dos de los ninos sometidos al tratamiento demostraron irritabilidad e inquietud durante la administración de Cortisona. En los restantes no se observó cambio en su comportamiento motor.

d) Complicaciones infecciosas durante el tratamiento: Durante la hormonoterapia se comprobó diarrea sin compromiso general en 4 niños igual que en el grupo control. Trastornos respiratorios agudos se presentaron en 3 opartunidades en ambos

$$
\text { ('TAHRO) XI } 7
$$

\section{AUMENTO PONDERAL EN PREMATUROS TRATA-}

\begin{tabular}{|c|c|c|c|c|}
\hline \multicolumn{3}{|c|}{$\begin{array}{c}\text { Edad ed dias } \\
\text { (promedio) }\end{array}$} & \multicolumn{2}{|c|}{$\begin{array}{c}\text { Aumento ponderal } \\
\left(\mathrm{B} T \mathrm{~s}_{\text {. }}\right)\end{array}$} \\
\hline & & & Tratados & No tratados \\
\hline $\begin{array}{r}9.3 \\
19.5 \\
29.5\end{array}$ & $\begin{array}{l}\mathbf{a} \\
\mathbf{a} \\
\mathbf{a}\end{array}$ & $\begin{array}{l}19,5 \\
29,5 \\
34,5\end{array}$ & $\begin{array}{r}(11) \\
121 \\
2 \times 3\end{array}$ & $\begin{array}{r}85 \\
161 \\
305\end{array}$ \\
\hline Tutal de de & & uttur en & $5: 464$ & 551 \\
\hline
\end{tabular}
DOS $Y$ NO TRATADOS CON CORTISONA. 


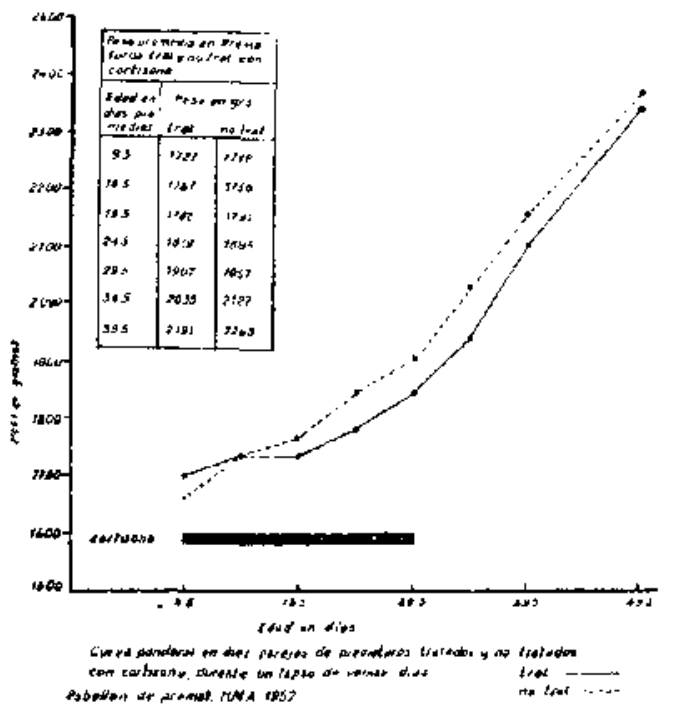

Grafico $N^{a}$ 1.

grupos. Abscesos subcutáneos se comprobaron en 2 niños tratados, coincidiendo con el sitio de la inyección de la hormona (cuadro 6).

\section{COMENTARTO}

Hemos tratado de aprovechar la experiencia obtenida en un corto número de prematuros tratados durante las primeras 6 semanas de vida, comparando cada uno de los pacientes con un prematuro de edad y peso semejantes, para observar de este modo si el tratamiento con Cortisona podría beneficiar desde el punto de vista clínico y principalmente de la evolución ponderal a los prematuros.

Los resultados obtenidos sobre la curva de peso no fueron muy importantes. Durante el período de tratamiento con Cortisona en nuestra serie de 10 niños, se obtuvo una ganancia ponderal diaria de 9 gramos (en el promedio); en tanto que en el grupo control, este aumento promedio fué de 12 gramos diarios. Esta diferencia es escasa $\mathrm{y}$ en ningún momento puede atribuirse al uso de Cortisona, ya que el número de casos que compone la serie estudiada no permite demostrar ni negar la influencia de la hormona. Si bjen en ambos grupos el ascenso ponderal fué mucho mayor después del $29,5^{\circ}$ día de vida, tampoco es significativo el discreto menor aumento ponderal en el periodo de 10 días comprendido desde el término del tratamiento en el grupo que recibió Cortisona en comparación con la ganancia en igual período de los niños no tratados (gráficos 1 y 2). Es probable que el tuso de mayores dosis hubiera permitido demostrar mayores diferencias (cuadro 7).

Nuestra impresión es que, si bien las diferencias ponderales no son significatjvas, durante el tratamiento fué cuando se observó una mayor separación de ambas curvas (gráficos 1 y 2 ', a la vez que el ascenso fué más elevado una vez suspendida la Cortisona, coincidiendo con las observaciones de Levine y Silvermann respecto a la detención del progreso ponderal durante las series hormonales y los mayores ascensos obtenidos posteriormente. En todo caso seria necesario hacer una
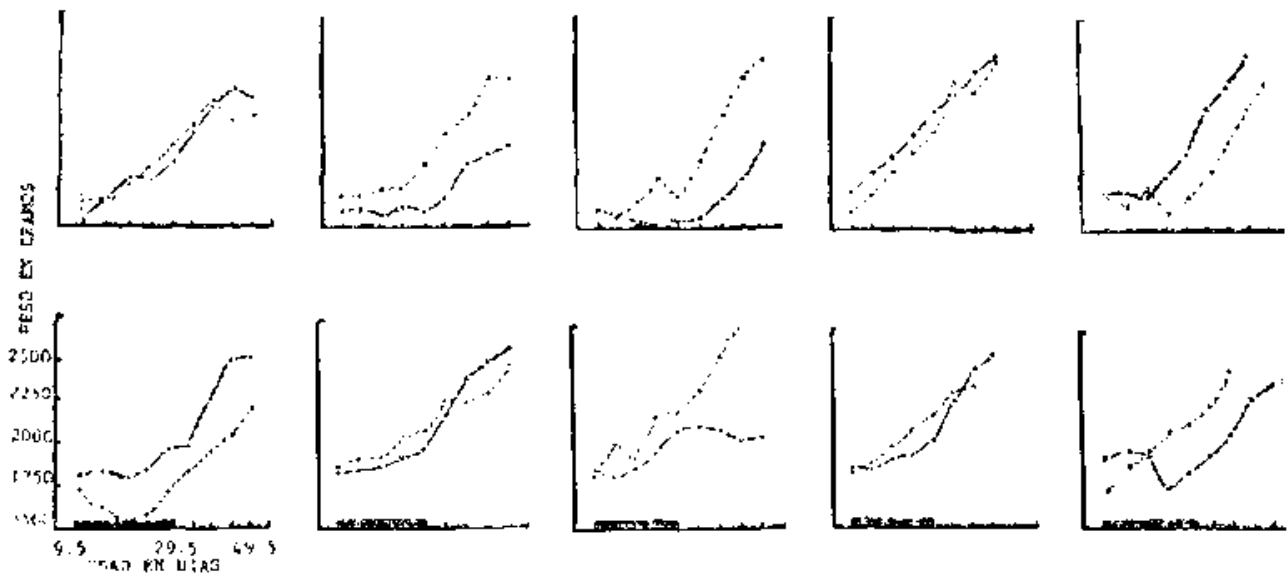

Gráfico Ne 2.

Curva ponderal en 10 parejas de prematuros tratados en cortisona docante un lapso de 20 días.

Tratados:

No tratados: ....... 
investigación semejante, pero mucho más amplia, en Servicios especializados de prematuros, hasta probar en forma definitiva el verdadero valor de la hormonoterapia con ACTH y Cortisona, ya sea, con cantidades diferentes a las que hemos utilizado o bien con nuestra dosificación.

A diferencia de lo que ocurre en el distrófico" sobre el apetito tampoco hubo acción aparente, por cuanto, no se observó aumento notorio de él en la mayoria de los niños y en uno hubo disminución franca que se atribuyó a un cuadro infeccioso sobre-agregado. En todo caso la respuesta al tratamiento en los 10 niños no fué uniforme y en relación al grupo control no hubo diferencias.

En cuanto a la mayor actividad física que otros autores han observado con el suministro de ACTH, tampoco podemos pronunciarnos. La inquietud e irritabilidad (demostrada por llanto y agitación) vista en 2 niños tratados, es un fenómeno que hemos podido también comprobar a menudo en niños no tratados con Cortisona.

Las complicaciones infecciosas fueron semejantes en su calidad y frecuencia en ambos grupos.

Aparte de estos resultados clínicos, la Cortisona nos pareció bien tolerada por los pacientes a las dosis usadas. Solamente en 2 casos se observó facies lunar al finalizar el tratamiento. Lo que atribuímos a la dosis usada que al parecer fué pequeña.

\section{RESUMEN}

Se tratan 10 prematuros con Cortisona durante un periodo de 20 dias. La dosis total usada fué de $180 \mathrm{mgrs}$. Se analiza la evolución clínica, morbilidad y curva de peso en relación a un grupo de control formado por 10 prematuros con igual peso de nacimiento y edad y pesos similares al iniciar la observación que duró 30 días en total. No se observaron resultados que pudieran atribuirse a la hormonoterapia. La escasa diferencia entre los pesos de ambos grupos se observó durante la hormonoterapia y aunque no es estadística- mente significativa coincide con las observaciones de otros autores, que durante el suministro de series hormonales de ACTH, abservaron una detención o disminución del ascenso ponderal en prematuros.

Es posible que un dosaje más elevado de la Cortisona o la administración de un mayor aporte calórico durante la hormonoterapia pudieran haber condicionado una evolución ponderal de otro tipo.

Sugerimos el interés de realizar una experiencia que contemple esas premisas.

\section{SUMMARY}

10 Prematures were treated with Cortison for a period of 20 days. The total dosis used was $180 \mathrm{mgrs}$. Clinical evolution, morbility and weight curve are analyzed in relation with a control group of 10 prematures with same birth weight, age and similar weights at the beginning of the experience which lasted 30 days. No results attributable to hormonal treatment were observed. A little difference between the weights of both groups was observed during the administration of Cortison and although this isn't statiscally significant coincides with other authors observations, that during the treatment with ACTH, a stop or decrease of the weight curve in prematures is seen.

It is possible that a higher dosage of Cortison or the administration of a greater caloric intake during the experience might have conditioned another type of weight curve.

It is of interest to work along these lines.

\section{BIBLIOGRAFÍA}

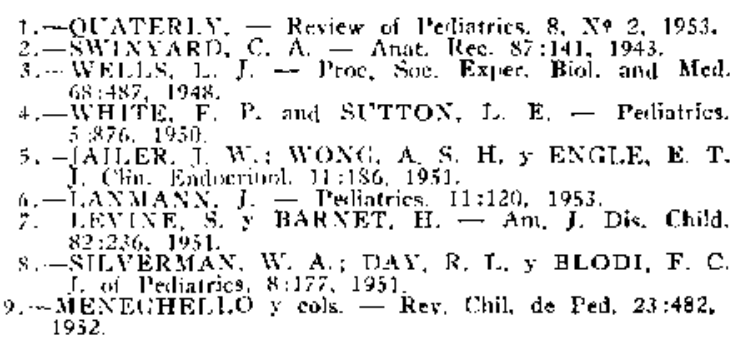

